\title{
Acerca del concepto de ejército industrial de reserva en la teoría de la dependencia ${ }^{[1]}$
}

\author{
On the concept of reserve army of labor in dependency theory
}

Recibido: 15/08/2021 Aprobado: 23/09/2021

\author{
Gil Felix ${ }^{[2]}$ \\ Universidade Federal da Integração Latino-americana (Brasil) \\ DOI: https://doi.org/10.29166/csociales.vli43.3209 \\ ORCID: https://orcid.org/0000-0001-8371-1358
}

\section{Resumen}

El artículo analiza el concepto de ejército industrial de reserva en la teoría propuesta por Ruy Mauro Marini en «Dialéctica de la dependencia» (1973) y en los artículos publicados complementariamente por el autor. En un ejercicio de revisita a los textos, analiza cómo se insertó este concepto en su esquema teórico y explica cuáles fueron los procesos por los que atribuyó especificidad al mismo en el ámbito de la llamada teoría marxista de la dependencia. Como conclusión, indica la centralidad de este tema en la teoría de Marini y propone un enfoque atento de esta premisa para los investigadores que analizan la superexplotación del trabajo actualmente a partir de su legado teórico.

Palabras claves: Ejército de reserva, Ruy Mauro Marini, dependencia.

\begin{abstract}
The article analyzes the concept of reserve army of labour in the theory proposed by Ruy Mauro Marini in «Dialéctica de la dependencia» (1973) and in complementary articles published by the author. Since the revisit to these texts, it analyzes how this concept was inserted in its theoretical scheme and explicit what processes were the ones by which he attributed specificity to it within the so-called Marxist dependency theory. In conclusion, it indicates the centrality of this topic in Marini's theory and proposes an attentive approach to this premise for researchers who analyze the super-exploitation of labor currently based on its theoretical legacy.
\end{abstract}

Key words: Reserve army of labour, Ruy Mauro Marini, dependency.

1 Una primera versión de este texto fue desarrollada a lo largo de una pasantía de investigación en el Centro de Estudios Latinoamericanos de la Facultad de Ciencias Políticas y Sociales de la Universidad Nacional Autónoma de México (UNAM), con beca de la Fundación de Amparo a la Investigación del Estado de San Pablo (Fundaçẩo de Amparo à Pesquisa do Estado de Säo Paulo-FAPESP), y publicada en portugués en Felix (2019).

2 Profesor e investigador del Instituto Latinoamericano de Economía, Sociedad y Política de la Universidade Federal da Integração Latino-americana (UNILA, Brasil). Autor de los libros O caminho do mundo: mobilidade espacial e condiçäo camponesa em uma regiäo da Amazônia Oriental y Mobilidade e superexploraçẩo do trabalho: o enig'ma da circulaçâo. Investiga temáticas relacionadas con ejército de reserva, movilidad, condición campesina, circulación y superexplotación del trabajo.

Los contenidos pueden usarse libremente, sin fines comerciales y siempre y cuando se cite la fuente. Si se hacen cambios de cualquier tipo, debe guardarse el espíritu de libre acceso al contenido. 


\section{Introducción}

En la sociología latinoamericana, el debate que entablaron Fernando Henrique Cardoso y Ruy Mauro Marini a finales de los años 1970 se caracterizó por una serie de controversias y, una vez abortado por Cardoso, terminó dejando muchos puntos abiertos. Uno de ellos es en relación a la naturaleza y al origen del régimen de superexplotación del trabajo que, según Marini, caracteriza las formaciones dependientes. En gran parte, Cardoso y Serra (1978) dedicaron un largo trecho de su texto crítico en torno al debate del intercambio desigual y, debido a eso, todavía hoy, es común las relecturas de las controversias teóricas de la cuestión más amplia de las interpretaciones marxistas de la transferencia de valor y de la dependencia. ${ }^{[3]}$ Sin embargo, más allá de esa cuestión, la respuesta de Marini a los críticos también reafirmó:

[...] la superexplotación del trabajo es acicateada por el intercambio desigual, pero no se deriva de él, sino de la fiebre de ganancia que crea el mercado mundial, y se basa fundamentalmente en la formación de una sobrepoblación relativa. Pero, una vez en marcha un proceso económico sobre la base de la superexplotación, se echa a andar un mecanismo monstruoso, cuya perversidad, lejos de mitigarse, es acentuada al recurrir la economía dependiente al aumento de la productividad, mediante el desarrollo tecnológico. (Marini, 1978, pp. 63-64; la bastardilla es mía)

Esa base a la que se refiere Marini fue poco analizada y constituye uno de los puntos abiertos del debate realizado con Cardoso, Serra y otros. ${ }^{[4]} \mathrm{Y}$, faltando mayores sistematizaciones de la relación entre el régimen de superexplotación del trabajo y la formación de una sobrepoblación relativa, muchos entusiastas recurren a teorizaciones ajenas, extrañas $\mathrm{y}$, a veces, hasta incluso contradictorias a la propuesta de Marini, en especial, cuando pretenden dar cuenta del análisis de cuestiones inherentes a la sobrepoblación relativa o ejército industrial de reserva (EIR) en las formaciones dependientes. Sin embargo, aunque Marini no haya sistematizado las observaciones respecto al ejército industrial de reserva en un texto específico, señaló en varias ocasiones su importancia y centralidad para el esquema teórico que proponía, como es el caso del trecho citado más arriba y de otros que serán mencionados en este artículo, principalmente de Dialéctica de la dependencia (1973) y de textos complementarios publicados por él en el mismo periodo.

En este texto, mi tesis principal es demostrar que el concepto de ejército de reserva tiene centralidad en la teoría de Marini, lo que será presentado a continuación, y la tesis secundaria, que se desprende de lo que fue expuesto más arriba, es que tal démarche coloca una perspectiva crucial para que se desarrolle actualmente el análisis del modo de producción capitalista a partir de su legado teórico. No obstante, como indiqué, tal tesis es todavía poco percibida, ofuscada y confundida, incluso entre los expertos. Siendo así, el texto también objetiva subsidiar teóricamente el trabajo de investigadores dedicados al estudio de la dependencia, así como de aquéllos que se han dedicado a investigaciones de situaciones concretas, entre los cuales me incluyo, junto a otros colegas reunidos en el Grupo de Investigación sobre Trabajo (Grupo de Pesquisa sobre Trabalho), actualmente con sede

3 En este texto, Cardoso y Serra (1978) critican a Marini. Las principales tesis de Marini fueron publicadas en Dialéctica de la dependencia (1973), Subdesarrollo y revolución (1974 [1969]) y El reformismo y la contrarrevolución (1976).

4 Amaral y Carcanholo (2008) es una excepción de abordaje exploratorio en ese sentido, orientado fundamentalmente a la explicación de la transferencia de valor. Antes de él, y paralelamente a Marini, Osorio (1979) también buscó analizar la relación entre superexplotación y ejército de reserva. Castro (1979), cuya tesis fue enfocada hacia el análisis del denominado comportamiento político del ejército de reserva, rememoró, en entrevista reciente, influencias teóricas diversas, mientras, que con Marini, que fue su tutor, habría armado el material empírico (Guimarāes, 2014, p. 229). 
en la Universidad Federal de la Integración Latinoamericana, en Brasil. ${ }^{[5]}$

Cabe, en este sentido, mencionar la opción propuesta a fin de buscar mayor fidelidad a las categorías utilizadas por el propio autor en los textos que serán citados, incluso cuando, a primera vista, pueda confundir a algunos lectores no especialistas, como fue el caso, por ejemplo, de la referencia a las «estructuras precapitalistas». Es conveniente aclarar a los lectores que Marini refutó enfáticamente la noción de "precapitalismo" en América Latina, así como también fue un crítico tenaz de los ya entonces denominados mitos del feudalismo y de la burguesía nacional, en defensa del carácter socialista de la revolución continental (como en, entre otros, Marini, 2008 [1973]; 2008a; 1974; s. f.).

En un momento en el cual, el concepto de ejército de reserva es pensado en dimensiones mundializantes y en el que la distancia, hasta entonces bien demarcada entre las morfologías de la clase trabajadora en las formaciones dependientes y en las centrales, parece disminuir con los procesos globales de precarización del trabajo que derivan de las transformaciones en la rotación del capital y en sus aspectos espaciales de desplazamiento, teorías anteriormente delimitadas en el ámbito de las ciencias sociales latinoamericanas ganan mayor adhesión y mayor poder explicativo (Felix, 2019).

No por casualidad, las tesis de la dependencia, y particularmente de Marini, han sido objeto de interés renovado en el mundo anglófono - tal como lo demuestran publicaciones recientes como Smith (2016), Sotelo (2016) y Kufakurinani et al. (2017) - y, por otro lado, el debate acerca del concepto de superexplotación del trabajo ha vuelto a la superficie en América Latina, a partir de la objeción o revisión de algunos economistas y de sus respectivos replicadores en los últimos años (Carcanholo, 2013; Osorio, 2013; Katz, 2017; Sotelo, 2017; Osorio, 2017). Sin embargo, se trata de un debate todavía poco orientado hacia la relación necesaria e intrínseca, aquí subrayada a partir de los textos del propio Marini, en principio, para el análisis de las formaciones dependientes y de lo que las mismas tendrían como especificidad estructural.

\section{Acumulación de capital y $\mathrm{EIR}$ (en general y en la formación social dependiente)}

El concepto de EIR o sobrepoblación relativa fue formulado por Marx en el libro I de $E l$ capital para dar cuenta de un fenómeno que está directamente relacionado con la acumulación de capital. ${ }^{[6]}$ Marini desarrolla y analiza las formas que asume el EIR en una determinada situación concreta específica, es decir, en las formaciones sociales dependientes, que se caracterizarían por el hecho de que, debido a las condiciones de su formación y desarrollo histórico, «agrava hasta el límite las contradicciones inherentes a la producción capitalista» (Marini, 1978, p. 102). Por lo tanto, antes de enumerar esas características específicas, cabe explicar cómo se forma y se reproduce socialmente el EIR de manera general.

En el capítulo 23 de El capital, Marx examina «la influencia que el aumento del capital ejerce sobre el destino de la clase trabajadora» (Marx, 2013, p. 689). Su factor más importante es la composición del capital y las

5 Tal como, dentre outros, Felix (2021a; 2021b; 2020; 2019; 2018); Felix y Sotelo (2019); Felix y Guanais (2018).

6 Marx trata los dos términos como sinónimos en El capital [1867], texto en que los conceptúa, revisa y publica originalmente. Sin embargo, actualmente, hay debates al respecto del origen y de la historia de la elaboración del concepto, así como del origen de la expresión que él utiliza para nominar a ese concepto (Felix, 2019). Actualmente, se sabe que Marx ya se ocupaba de la cuestión en manuscritos no publicados desde 1847, y Engels, desde su libro de 1845 (Engels, 2010). Y que ambos se habrían inspirado en categorías creadas en el seno del propio movimiento operario de la época. Marini utilizó la forma que aquí abrevié en la sigla EIR. En sus principales textos, publicados en castellano, él cita referencias de la traducción de El capital de la editora Fondo de Cultura Económica y de las ediciones francesas de los textos de Marx bajo dirección de Maximilien Rubel. 
variaciones que la misma sufre a lo largo del proceso de acumulación. Marx demuestra, en ese sentido, que hay una disminución relativa del capital variable (parte que se refiere al valor de la fuerza de trabajo) en la composición del capital, a medida que avanzan la acumulación y la concentración que la acompaña. Cuanto mayor acumulación, mayor el desarrollo de la productividad del trabajo social, mayor el cambio en la composición del capital, en términos relativos de valor, con mayor importancia de su parte constante en detrimento de su parte variable.

Así, la acumulación capitalista produce constantemente lo que Marx denominó EIR, una parcela de la clase trabajadora que se torna excedente en relación al capital, es decir, relativamente excedente para las necesidades de valorización del capital. Según Marx, en el caso del capitalismo, la verificación de una población excedente es tanto un producto necesario de la acumulación de capital como una palanca de esa misma acumulación y «[...] hasta incluso una condición de existencia del modo de producción capitalista» (Marx, 2013, p. 707).

La producción y la existencia de un ejército de reserva, dada la competencia, disciplina de los regímenes de trabajo del ejército activo de trabajadores, ya sea en el sentido del capitalista de exigir la intensificación y/o la prolongación de las jornadas laborales, ya sea en el sentido de imponer el precio de la fuerza de trabajo, el salario u otros dictámenes del capital.

Para Marini, el análisis del capitalismo en América Latina lleva a notar que ocurre lo que él denomina un capitalismo sui generis, "que solo tiene sentido si lo contemplamos en la perspectiva del sistema en su conjunto, tanto a nivel nacional como, y principalmente, a nivel internacional» $\left(\mathrm{Ma}^{-}\right.$ rini, 2008, p. 108). O sea, es en las condi- ciones colocadas por el mercado mundial que el mismo se forma y desarrolla, histórica y estructuralmente, las principales características que lo distinguen. En este sentido, es el conocimiento de la forma particular que tomó el capitalismo latinoamericano lo que permite comprender su gestación y las tendencias que lo llevaron hasta esta formación en la que se presenta, dependiente. Los fenómenos por los cuales se distingue no son nada más que manifestaciones de la manera particular de cómo incide, en esa formación, la ley general de la acumulación de capital. Y, tal como Marini desarrolla en Dialéctica de la dependencia, el fundamento de la dependencia es la superexplotación del trabajo. Es la superexplotación el fenómeno que fundamenta la formación de la economía exportadora y, después, determina el desarrollo de la economía industrial dependiente en sus diversas fases y en su expansión productiva.

No obstante, a su vez, la base de un régimen de superexplotación del trabajo es, como afirma Marini, un «inmenso ejército industrial de reserva» que caracteriza las formaciones sociales dependientes y las singulariza frente a otras formaciones. Por lo tanto, cabe comprender lo que sería un EIR que se forma y se reproduce socialmente con determinadas especificidades colocadas por la formación social dependiente o lo que yo, en adelante, adjetivo como un EIR ampliado. ${ }^{[7]}$

En el caso de la economía dependiente, es la existencia del EIR que sustenta el régimen de la superexplotación del trabajo, así como, al mismo tiempo, la existencia de este régimen establece la formación de un EIR ampliado. La superexplotación del trabajo se caracteriza por la intensificación del trabajo, por la prolongación de la jornada de trabajo y por el vilipendio del fondo de consumo del trabajador, quiere decir, por la reproducción

7 En este caso, la noción de EIR expandida no debe confundirse con las formas del ejército de reserva ya descritas por Marx (2013). El EIR ampliado se presenta en todas las formas descritas por Marx en formaciones sociales dependientes (líquida o fluida; latente; estancada). 
de un régimen o sistema de valor que dilapida la fuerza de trabajo del trabajador.

Para Marini, la existencia de un «inmenso EIR» es la base de ese régimen, en la medida en que da las condiciones para la imposición del mismo al conjunto de la clase trabajadora en una formación social dependiente. Por otro lado, dadas las singularidades de ese régimen, al superexplotar la fuerza de trabajo del ejército activo de trabajadores, se forma y se reproduce un EIR en las enormes proporciones y con las características que se presentan en la periferia capitalista. Hay, sin embargo, procesos que llevan a la producción de ese EIR ampliado en las formaciones sociales dependientes. Voy a enumerarlos a continuación, revisitando algunos textos de Marini.

\section{a. Papel del capital extranjero}

En «El ciclo del capital en la economía dependiente» (1979), Marini demuestra de qué maneras se manifiesta la dependencia en relación al exterior en las economías dependientes y el carácter que asume la producción interna a partir de eso. Por tanto, él analiza las tres fases del ciclo del capital: circulación, producción y circulación nuevamente, sintetizados en la fórmula $\mathrm{D}-\mathrm{M}$... P ... M' - D'. En la primera fase de circulación, el capital dinero toma la forma de mercancías (medio de producción y fuerza de trabajo). En la economía dependiente, ese capital dinero tiene tres fuentes: el capital privado interno, la inversión pública (Estado) y el capital extranjero. Entre ellas, asumen gran importancia la inversión del Estado y el capital extranjero. Nos interesa aquí principalmente el capital extranjero, que se presenta bajo dos formas: como inversión directa (forma que era predominante en América Latina hasta la década de 1960) y como inversión indirecta, que es cuando el capital extranjero se pone a disposición de los capitalistas internos (nacionales y extranjeros) bajo la forma de préstamos o financiaciones - manera por la cual el mismo deja de asumir los riesgos de la producción y realización de la plusvalía-. Es importante colocar que el capital extranjero se mueve tanto en el sentido de entrada en la economía dependiente como en el de salida, en la forma de transferencias de plusvalía para el exterior. En la medida en que se incorpora en esa fase, el capital extranjero, factor externo a la economía dependiente y totalmente fuera de su control, se internaliza y se constituye como un factor directo del ciclo del capital, del cual depende el proceso de acumulación y producción y el restante del ciclo.

Importa destacar que esa economía depende del flujo externo de capital, así como también depende, para completar la primera fase de circulación, de medios de producción adquiridos en el exterior, lo que hace que el ciclo del capital sea, como afirma Marini, doblemente articulado y doblemente dependiente del exterior. Parte de los medios de producción vienen del exterior, particularmente equipos y maquinarias. Eso ocurre en cualquier economía, sin embargo, en la economía dependiente, gana un contorno especial, debido a que su industrialización no es «orgánica», quiere decir, la industria de bienes de consumo no tiene como consecuencia el desarrollo de la industria de bienes de capital. Según Marini, la economía dependiente no cuenta con un sector dinámico de bienes de capital, que tiene origen en buena parte en el sector de bienes de capital de los países avanzados, adquiridos, por lo tanto, del exterior, vía mercado mundial. Y esa dependencia no es solo de equipos, sino también del conocimiento necesario para operarlos y, cuando es el caso, de la tecnología para fabricarlos.

Entre otras consecuencias, la inserción de esa maquinaria y tecnología, desarrolladas para una economía formada bajo otro patrón de producción y productividad, lleva a una 
rápida expansión del EIR en la economía dependiente. Las nuevas técnicas de producción insertadas son construidas en contextos en que hay una búsqueda por mayor productividad de la fuerza de trabajo y reducción del número de trabajadores empleados.

La segunda fase del ciclo de acumulación y producción se desarrolla a partir de la primera: las empresas extranjeras o asociadas, con acceso a una tecnología más avanzada, operan con medios de producción que les garantizan supremacía en relación a las empresas nacionales, así obtienen una plusvalía extraordinaria y concentran cada vez más de lo que invierten en la economía dependiente. Las empresas medianas y pequeñas, que operan en condiciones medias de producción (o por debajo de eso) y transfieren parte de la plusvalía para las empresas monopólicas, reaccionan a esa situación recomponiendo su tasa de ganancia a través de la elevación de la tasa de plusvalía, obtenida a costo de la extracción de más trabajo no remunerado de sus trabajadores y sin variación significativa en la productividad, o sea, a través de mecanismos de superexplotación del trabajo. A su vez, los capitales monopólicos también se encargan de emplear trabajadores con remuneración similar a las demás empresas, con salarios inferiores al valor de la fuerza de trabajo, y se completa, en ese sentido, un círculo vicioso.

Por lo tanto, la producción es realizada en condiciones de superexplotación del trabajo y con base en la rápida creación y expansión de un EIR que crece a partir de características inherentes al ciclo del capital en la economía dependiente, en especial, como ya fue dicho, a partir de la forma en la que incide la inversión extranjera. La superexplotación del trabajo, por un lado, comprime el ejército activo y lo hace crecer lentamente, exigiendo más trabajo de la parte ocupada de la clase trabajadora. Por otro lado, el capital extranjero, del cual depende el ciclo del capi- tal desde el inicio, promoviendo la inserción de tecnologías y maquinarias «parcas en mano de obra», forma, por esos dos factores, un EIR ampliado. En la economía dependiente, el aumento de la productividad expande de forma radical el EIR. Aquí, como en todos los demás aspectos, la formación dependiente revela una vez más su peculiaridad que, tal como describe Marini, «corresponde a la agudización hasta el límite de las contradicciones inherentes al modo de producción capitalista» (Marini, 1979, p. 53).

\section{b. Aumento de la productividad}

Como fue dicho más arriba, el aumento de la productividad en las economías dependientes se corresponde con una expansión radical del EIR. Y, cabe mencionar, el aumento de productividad no es incongruente con el régimen de superexplotación del trabajo que, debido a las características descriptas anteriormente, obstaculiza el pasaje de la producción de plusvalía absoluta para el de plusvalía relativa, como forma hegemónica de extracción de plusvalía en las formaciones dependientes. Al contrario de lo que ocurre en el desarrollo histórico del capitalismo clásico en los países avanzados, la producción de plusvalía relativa no se convirtió en dominante en las relaciones entre capital y trabajo en las formaciones dependientes, considerando que la superexplotación del trabajo, fundamento de la dependencia, es el régimen predominante. Como ya se describió, el aumento de la productividad en la producción realizada en la economía dependiente no objetiva una producción de plusvalía basada en la rebaja del valor de la fuerza de trabajo, sino el aumento de la masa de valor producida, una vez que esa producción se vuelve para artículos que no entran, o entran muy escasamente, en el consumo individual de los trabajadores que, por otro lado, están sujetos a un régimen que aprecia por la intensificación del trabajo, por el aumento de la 
jornada de trabajo y por la disminución indefinida de los salarios.

No hay ni hubo, como mucho ya se debatió al respecto de la teoría formulada por Marini (2008; 2008a), ausencia de aumento de la productividad en la economía dependiente, aunque, en ese sentido, tal aumento de productividad se desarrolle en condiciones de superexplotación del trabajo, o sea, no se confunda necesariamente con producción de plusvalía relativa, que es lo que ocurre en los países avanzados, como mínimo desde el gran periodo de la Revolución Industrial en Inglaterra (considerando que, junto con las transformaciones técnicas del sistema productivo, los trabajadores fueron paulatinamente incorporados en el consumo de los bienes producidos por las fábricas de la gran industria).

En ese sentido, en Dialéctica de la dependencia (2008; 2008a) Marini insiste en diferenciar los conceptos de productividad y de plusvalía relativa. Él explica que una mayor capacidad productiva del trabajo no significa necesariamente aumento de la plusvalía relativa, aunque sea una condición primordial para que la misma ocurra. Al aumentar la productividad, el trabajador crea más productos en el mismo tiempo, pero no más valor. Con eso, el capitalista logra rebajar el valor individual de sus mercancías en relación al valor social, es decir, el que se le atribuye debido a las condiciones generales de la producción, y así consigue una plusvalía superior a los demás capitalistas, una plusvalía extraordinaria. La búsqueda por la apropiación de esa plusvalía, de una ganancia extraordinaria, es, en verdad, lo que mueve al capitalista. Pero eso no modifica la tasa de plusvalía, el grado de explotación del trabajo en la economía, lo que apenas ocurre si hay cambio en la relación entre el tiempo de trabajo excedente y el tiempo de trabajo necesario a favor del capitalista, o sea, si se reduce el tiempo en que el trabajador reproduce el valor de su fuerza de trabajo. Y, para que el valor de la fuerza de trabajo sea disminuida, es necesario que la desvalorización de las mercancías producidas incida sobre aquéllas que componen el conjunto de los bienes necesarios para la reproducción de la fuerza de trabajo, los bienes-salario. Apenas cuando se rebaja el valor de los bienes-salario hay cambio de la tasa de plusvalía y, por lo tanto, producción de plusvalía relativa. Siendo así, el aumento de la productividad provocaría esa desvalorización de los bienes-salario, pero eso no ocurre necesariamente. $\mathrm{Y}$ es justamente eso lo que hace que, en las economías dependientes, por ejemplo, haya producción de plusvalía extraordinaria y, en ese sentido, aumento de la intensidad y de la productividad del trabajo, sin que haya, predominantemente, producción de plusvalía relativa. Marini, en conclusión, escribe que ese es el problema de fondo que la teoría marxista de la dependencia está urgida a enfrentar, es decir:

[...] el hecho de que las condiciones creadas por la superexplotación del trabajo en la economía capitalista dependiente tienden a obstaculizar su tránsito desde la producción de plusvalía absoluta a la de plusvalía relativa, en tanto que forma dominante en las relaciones entre capital y el trabajo. La gravitación desproporcionada que asume en el sistema dependiente la plusvalía extraordinaria es un resultado de esto y corresponde a la expansión del ejército de reserva y al estrangulamiento relativo de la capacidad de realización de la producción. Más que meros accidentes en el curso del desarrollo dependiente o elementos del orden de la transición, estos fenómenos son manifestaciones de la manera particular de cómo incide en la economía dependiente la ley general de la acumulación de capital. En última instancia, es de nuevo a la superexplotación del trabajo que tenemos que referirnos para analizarlos. (Marini, 2008a, p. 163)

La dificultad para generalizar la plusvalía relativa no significa ausencia de incremento en la productividad del trabajo en las economías dependientes. Y, como ha sido verificado en 
el proceso de industrialización de algunos de esos países, muy por el contrario. Tal como lo descrito por Marini (2008a), el aumento de productividad se conjuga con la superexplotación del trabajo, que es, incluso, posibilitada por él mismo, en la medida en que la producción capitalista no suprime los mecanismos de mayor dilapidación de la fuerza de trabajo del trabajador, sino que, por el contrario, los acentúa, siempre que alcanza una forma de producir más en menos tiempo, o en el mismo tiempo que antes. Hay una estrecha interdependencia entre el aumento de la productividad, la intensificación del trabajo y la duración de la jornada, cada cual posibilitando el incremento del otro.

A su vez, el aumento de la capacidad productiva del trabajo es, como ya describía Marx, expansión del EIR, una vez que ese aumento implica la reducción de la parte relativa a la fuerza de trabajo en la composición del capital. En las condiciones de formación social dependiente, tal tendencia se agrava al límite, pues el aumento de la productividad se desarrolla en medio de un régimen de superexplotación del trabajo, exigiendo más trabajo del ejército activo de trabajadores al mismo tiempo que expulsa trabajadores para la reserva. El aumento de la composición orgánica del capital, cuando es el caso, expresa mayor dilapidación de la fuerza de trabajo del ejército activo de los trabajadores (intensificación, aumento de la jornada) y no, necesariamente, incorporación de más fuerza de trabajo en término absolutos.

Son esas conclusiones al respecto del aumento de la productividad en las economías dependientes que le permiten a Marini hacer la crítica a las teorías de la marginalidad, entonces en boga en los años 1970, en especial de las tesis de José Nun y de Aníbal Quijano. La producción y la reproducción de un EIR ampliado se deben principalmente al modo en que incide el aumento de la productividad en las economías dependientes, que, como analiza Marini, se da de forma que acentúa la superexplotación de la fuerza de trabajo y no al contrario. Es en ese sentido que se debe comprender la enorme dimensión que toma el EIR en estas formaciones, quiere decir que, es de esa manera como se expresa la ley general de la acumulación capitalista en las sociedades latinoamericanas.

El primero [punto] es fundamental [la producción capitalista, al desarrollar la fuerza productiva del trabajo, no suprime, sino que acentúa la mayor explotación del trabajador], si se quiere entender cómo actúa la ley general de la acumulación capitalista, o sea, por qué se produce la polarización creciente de la riqueza y la miseria en el seno de las sociedades en que ella opera. Es en esta perspectiva, y solamente en ella, como los estudios sobre la llamada marginalidad social pueden ser incorporados a la teoría marxista de la dependencia; dicho de otra manera, sólo así ésta podrá resolver teóricamente el problema planteado por el crecimiento de la superpoblación relativa con las características extremas que presenta en las sociedades dependientes, sin caer en el eclecticismo de José Nun, que el mismo Cardoso criticó con tanta razón, ni tampoco en el esquema de Aníbal Quijano, que, independientemente de sus méritos, conduce a la identificación de un polo marginal en esas sociedades que no guarda relación con la manera como allí se polarizan las contradicciones de clase. (Marini, 2008a, p. 159)

El «eclecticismo de Nun» que, como escribe Marini, habría sido criticado por Fernando Henrique Cardoso «con tanta razón», se caracteriza por una teoría funcionalista al respecto del papel desempeñado por determinados grupos poblacionales frente a los sistemas de producción. ${ }^{[8]}$ No se refiere a una teoría de la acumulación, tal como expresaba Marx en El capital en la construcción de la noción de EIR, lo que hace que lo que Nun (2001) denomina «masa marginal» sea un concepto que no se inserta en ninguna aserción marxiana

8 La crítica a la que Marini se refiere está en Cardoso (2001). 
o marxista de la realidad social latinoamericana. Para Marini, la teoría de la marginalidad social apenas tendría sentido en el caso de que estuviese relacionada con la forma en la cual se desarrolla la acumulación en las sociedades dependientes, es decir, estuviera, por ejemplo, relacionada con los procesos que llevan al aumento de la fuerza productiva del trabajo y a la formación de un EIR específico en esas sociedades. Concluye Marini:

(...) según el grado de desarrollo de las economías nacionales que integran el sistema, y del que se verifica en los sectores que componen cada una de ellas, la mayor o menor incidencia de las formas de explotación y la configuración específica que ellas asumen modifican cualitativamente la manera como alli inciden las leyes de movimiento del sistema, y en particular la ley general de la acumulación de capital. Es por esta razón que la llamada marginalidad social no puede ser tratada independientemente del modo como se entrelazan en las economías dependientes el aumento de la productividad del trabajo, que se deriva de la importación de tecnología, y la mayor explotación del trabajador, que ese aumento de la productividad hace posible. No por otra razón la marginalidad sólo adquiere su plena expresión en los países latinoamericanos al desarrollarse en estos la economía industrial. (Marini, 2008a, 162, las bastardillas son del autor).

\section{c. Acentuación del proceso de concentración y centralización del capital}

Es necesario examinar todavía más detenidamente los efectos provocados por la llamada inversión «extranjera» en la teoría marxista de la dependencia. Es a partir de la misma que, en gran parte, se industrializan los principales países latinoamericanos, en especial, después de la década de 1940, como ya mencioné anteriormente. En La acumulación capitalista mundial y el subimperialismo (1977), Marini llama la atención para la acentuación del proceso de concentración y centralización del capital que la inversión acarrea, lo que, por regla general, siempre ocurre, pero, dadas las condiciones de la economía dependiente, termina asumiendo proporciones mucho mayores.

[...] esto [proceso de concentración y centralización del capital] acompaña la ampliación de escala de la acumulación capitalista, siendo un fenómeno natural; sin embargo, por las condiciones económicas de los países avanzados, en que son mayores los niveles tecnológicos y de capital mínimo exigido para la puesta en marcha de la producción, la inversión extranjera, al incidir en una economía más atrasada, provoca de golpe una fuerte concentración del capital y conduce prontamente a la centralización. (Marini, 1977, p. 26)

En el artículo en cuestión, Marini (1977) cita ejemplos, como era el caso de Chile en 1968, en donde, cerca del 3\% de la industria manufacturera controlaba el $44 \%$ de la ocupación, el $58 \%$ del capital y el $52 \%$ de la plusvalía generada en el total de la industria. Tales procesos habrían formado un gran capital extremamente superior frente al conjunto de la clase capitalista. Y, junto con ellos, una industrialización con baja creación de empleos que, sumados a fenómenos estructurales de las formaciones sociales latinoamericanas, generó un EIR de enormes proporciones. Hubo, en ese sentido, un gran desencuentro entre la búsqueda y la oferta de empleos:

Se ha asistido a un doble proceso: por un lado, las
formas de tenencia de la tierra y la introducción
de innovaciones tecnológicas en el agro, así como
las expectativas de empleo y salario provocadas
por la industria manufacturera, han generado
fuertes movimientos de migraciones internas y un
proceso acelerado de urbanización. Por otra parte,
en buena medida por la elevación del nivel tecno-
lógico, aunque también por limitaciones de la tasa
de inversión, la masa trabajadora se ha enfrentado
a crecientes dificultades para encontrar trabajo.
(Marini, 1977, p. 27) Marini (1977) también cita ejemplos, en este caso, el de Venezuela, que, en rápida industrialización en la posguerra vio la tasa de desempleo en los años 1950 duplicarse, del 6,2\% al 13,7\% 
Acerca del concepto de ejército industrial de reserva en la teoría de la dependencia

p-ISSN 0252-8681 | año 2021 | núm. 43 | pp. 123-0137

en 1960. Cita, además, que según la OIT (Organización Internacional del Trabajo), el denominado «desempleo disfrazado» o subempleo en las zonas urbanas de América Latina alcanzaría del 30 al 40\% de la fuerza de trabajo. ${ }^{[9]}$

\section{d. Absorción de las estructuras precapitalistas}

Otro factor relevante en la formación de un EIR ampliado es el proceso de absorción de las estructuras precapitalistas, que Marini califica como un «proceso lento» en las formaciones dependientes. En «Estado y crisis en Brasil» (1977a), Marini se refiere a los aspectos que hacen que haya una debilidad de la burguesía latinoamericana frente al Estado, dividida en un conjunto de clases o fracciones de clase presentes en las formaciones sociales dependientes. Al hacer esos comentarios en el artículo mencionado, Marini afirma que «aunque articuladas e integradas bajo la dominación del modo de producción capitalista, las estructuras precapitalistas son objeto de un proceso lento de absorción» (Marini, 1977a, p. 76).

Este proceso fue más evidente a lo largo de la industrialización latinoamericana, en todas sus contradicciones, siendo uno de los fenómenos más flagrantes el ya mencionado desencuentro entre las masas que buscaban empleos en la industria de las ciudades y la inexpresiva oferta de los mismos, aliada a los bajos salarios que eran ofrecidos. El resultado de ese proceso fue una formación urbana constituida por enormes bolsones de miserables, inmensas periferias y aglomeraciones con baja o ninguna estructura de servicios básicos como iluminación, saneamiento, asistencia de la salud, transporte, limpieza, etc. Junto a la expectativa de empleos y salarios, Marini cita otros factores que llevaron a la migración campo-ciudad y a la acelerada y precaria urbanización en los países dependientes, en especial, las formas de propiedad de la tierra, la ausencia de una reforma agraria y la introducción de las nuevas tecnologías en la agricultura. ${ }^{[10]}$

Por lo tanto, la industrialización de los años 1950 habría fomentado lo opuesto de lo que esperaba la Cepal (Comisión Económica para América Latina y el Caribe), como resumió Marini en un texto de 1989 recientemente publicado (Marini, 2012, pp. 197-198).

O sea, en la medida en que no existen reformas básicas y no se ofrecen empleos suficientes en las ciudades, el proceso de absorción de las estructuras precapitalistas en curso en América Latina significa la producción de un EIR ampliado en esas formaciones. Hay que considerar, en ese sentido, que, a medida que se desarrolla un proceso histórico lento en el cual cada vez más fracciones de esas poblaciones son desplazadas para las ciudades, hay un aumento del EIR, ya que la proletarización de esas poblaciones se da por medio de su transformación en EIR.

Además de eso, como discurre Marini en Dialéctica de la dependencia (2008), apenas con la proletarización se garantiza plenamente el régimen de superexplotación del trabajo. En este texto, Marini escribe respecto del proceso histórico de proletarización a fin de demostrar por qué otras relaciones de trabajo constituyen un obstáculo para la institución del régimen de superexplotación del trabajo, en la medida en que solo cuando hay sujetos «libres» y llevados a vender su fuerza de trabajo es posible tener un régimen en el cual el precio de esa mercancía es sistemáticamente mantenido por debajo

9 En el caso de Brasil, los conceptos utilizados en el Censo Demográfico oficial encubren una importante porción del EIR bajo la forma de desempleo disfrazado, así, por ejemplo, excluyen a las mujeres ocupadas en el hogar como «inactivas», como el autor también indica en un texto del mismo periodo (Marini, 1978, p. 86).

10 Debido a eso, antes, él ya había enfatizado el carácter socialista que toma la reforma agraria en la lucha de clases latinoamericana frente a los intereses congruentes entre la burguesía agraria y las demás fracciones burguesas (Marini, 1976a). En el caso de Brasil, incluso, tal análisis sería fundamental para caracterizar el golpe militar de 1964, en textos cuyas primeras versiones remontan a 1965 (Marini, 1974). 
de su valor. Cuando lo que es mercancía es la fuerza de trabajo (y no el trabajador), se le deja al trabajador el cuidado del tiempo no productivo, desde el punto de vista capitalista, se apropia apenas del tiempo de su existencia utilizable en la producción y se posibilita, con eso, la remuneración por debajo del valor.

Siendo así, es posible afirmar que, a partir de la teoría de Marini, «en las formaciones dependientes, además del movimiento ya descrito por Marx de disminución relativa del capital variable y aumento del EIR, hay también compresión del ejército activo de trabajadores (concentración y centralización del capital peculiares), un régimen de superexplotación del trabajo y un aumento en la productividad que, en estas condiciones, junto con un lento proceso de proletarización y absorción de las estructuras precapitalistas, redundan en un EIR ampliado». Ese EIR es, de acuerdo con el propio movimiento de acumulación de capital, parcialmente incorporado (y después descartado) en régimen de superexplotación del trabajo en el proceso productivo.

\section{EIR ampliado y superexplotación del trabajo}

La existencia de un EIR ampliado en las formaciones sociales dependientes posibilita y reproduce el régimen de superexplotación del trabajo que las caracteriza. Y lo hace a partir de una serie de dinámicas establecidas por su acción social. La amenaza a la situación del ejército activo de trabajadores y al debilitamiento de la capacidad reivindicativa de la clase como un todo son algunas de ellas.

En «El ciclo del capital en la economía dependiente» (1979), al analizar la fase de acumulación y producción del ciclo del capital, Marini hace referencia al EIR como un elemento fundamental para que pueda operar la superexplotación, o sea, como menciona, en cuanto un mecanismo por el cual el capital debilita la clase obrera (Marini, 1979, p. 50). En ese sentido, hay una dinámica política ejercida por el EIR en las formaciones dependientes, que crea las condiciones que las singulariza frente a otras formaciones. Es la presión de los trabajadores no ocupados, u ocupados de manera irregular, sobre el sector empleado de la clase que propicia que se mantenga el régimen. Junto a esa presión, el capital también recurre al Estado a fin de estipular procedimientos que hacen que la función cumplida por el EIR sea más eficaz, como es el caso de la facilitación de los despidos, contrataciones y recontrataciones o, como agrega Marini, entre otras medidas, la eliminación de la estabilidad laboral, la supresión del derecho de huelga y la fijación de techos salariales (Marini, 1979a, pp. 37-38).

La dictadura implantada en Brasil en 1964, por ejemplo, fue eficaz en ese sentido. Marini, en "Las razones del neodesarrollismo", cita la institución del Fondo de Garantía por Tiempo de Servicio (FGTS) como un mecanismo que facilitó la acción del EIR, al permitir «mayor rotación de la mano de obra» (Marini, 1978, pp. 87-88). Por lo tanto, con el FGTs, fue posible que las empresas en Brasil despidiesen a los trabajadores en vísperas de despidos colectivos y los readmitiesen después, o contratasen nuevos, por salarios más bajos que los establecidos en el acuerdo salarial. Además de eso, las indemnizaciones por tiempo de servicio que los trabajadores pasaron a recibir del FGTS eran menores que las que recibían por el mismo tiempo en el régimen anterior. La rotación propiciada por el nuevo régiimen también influyó, indirectamente, a nivel salarial, pues desorganizó a los trabajadores, privilegiando a los nuevos en detrimento de los más antiguos, cuya tasa de sindicalización, por ejemplo, era más elevada.

La presión del EIR ampliado también influye sobre el nivel de los salarios y posibilita que sean reducidos o instituidos salarios míni- 
mos por debajo del valor de la fuerza de trabajo. En «La acumulación capitalista mundial y el subimperialismo» (1977), Marini observa que la participación de los sueldos y salarios de los operarios en valor agregado del sector manufacturero es en Brasil la mitad de lo que representa en los Estados Unidos e Inglaterra. En "Las razones del neodesarrollismo", Marini llega a afirmar que la dictadura militar brasileña habría, incluso, fijado el salario real por debajo del valor de la fuerza de trabajo (Marini, 1978, p. 89). «El EIR ampliado, por lo tanto, actúa de forma tal que sostiene al régimen de superexplotación del trabajo, garantizando la tendencia de explotar al máximo la fuerza de trabajo del sector ocupado de la clase al mismo tiempo en que posibilita la reposición de esa fuerza de trabajo y permite la fijación de su precio por debajo del valor».

La acción del EIR ampliado se observa, en especial, en los momentos en que hay mayor incorporación de sus capas al ejército activo de trabajadores, como es el caso de los periodos en que la acumulación de capital está en ritmo acelerado y se emplea más fuerza de trabajo en el proceso productivo. Marini (1977) analiza el periodo comprendido entre 1956 y 1974 en Brasil, en el que hubo aumento en el número de trabajadores empleados - en particular de los que recibían hasta un salario mínimo- y demuestra que, junto con ese aumento, hubo decrecimiento en el valor del salario real. O sea, la incorporación del $\mathrm{EIR}$ se dio con reducción de salarios reales, incluso en el periodo del denominado «milagro económico» brasileño, entre 1968 y 1973. El EIR ampliado garantiza, en ese sentido, la incorporación de más fuerza de trabajo sin alterar el régimen de la superexplotación del trabajo, en especial, en los momentos en que hay crecimiento económico y aumento del ejército activo de trabajadores en las economías dependientes.

O sea, en una expresión límite de la ley general de acumulación formulada por Marx, la existencia de un EIR ampliado implica una relativa independencia entre los movimientos de la acumulación y las condiciones de salario derivadas de ese movimiento. La incorporación de mayores parcelas del EIR, incluso en los ciclos expansivos del capital y consecuente aumento en el ejército activo, no implica alteración en los niveles salariales. Son variables relativamente independientes en esas formaciones. Mediado por el EIR ampliado, el proceso de acumulación no altera los salarios. Considerando, con Marini (2008), que la acumulación en las economías dependientes depende más del aumento de la masa de valor que de la tasa de plusvalía, una vez más, se demuestra así que la dinámica de la acumulación en esas formaciones sociales poco se relaciona con el nivel de los salarios, mantenidos, por regla general, en condiciones de superexplotación del trabajo (por debajo del valor de la fuerza de trabajo).

Marini cree que la singularidad con que se presenta el capitalismo dependiente se debe a la forma particular como incide en el mismo la ley general de la acumulación, teniendo las contradicciones del modo de producción capitalista aguzadas al límite. La formación y la reproducción del EIR ampliado, es decir, un EIR con características específicas de las formaciones dependientes, tal cual fue descrito en el texto, por lo tanto, expresa esas contradicciones en la base del régimen que las fundamenta.

\section{Comentarios finales}

Después de una breve introducción con consideraciones acerca del legado teórico de Ruy Mauro Marini, de la construcción del objeto de este artículo y su relevancia, este texto fue organizado en dos subdivisiones. En la primera, expuse el concepto de ejército industrial de reserva de Marx en la tesis de Marini y, a 
continuación, detalladamente, los procesos que se constituyen en la formación social dependiente, lo que sugerí denominar EIR ampliado. En la sección siguiente, con ejemplos tomados a partir de artículos de Marini, analicé la relación entre este ejército de reserva y el régimen de superexplotación del trabajo, resumiendo lo que sería una tesis general en el ámbito de su teoría al respecto de la ley general de acumulación capitalista en condiciones dependientes.

Al dar cuenta de la lógica de la acumulación en América Latina, el tema principal que refiero en esta revisión es que implica, fundamentalmente, un análisis de las condiciones sociales de la lucha de clases, que es quizás lo que mejor representa el legado teórico marxista de Marini para la dependencia distante de otros/as autores e investigaciones que poco lograron superar las limitaciones ideológicas burguesas de las corrientes teóricas opuestas exagerando o enfatizando exclusivamente las relaciones de intercambio en detrimento de las relaciones de producción y enfocándose en el tema del llamado desarrollo nacional o «asociado» en detrimento de explotación/ dominación de clases.

En este sentido, el texto aclara y sistematiza el abordaje de Marini, que comprendía, a partir de Marx, las especificidades latinoamericanas en el ámbito del universo dialéctico de la acumulación de capital, de la lucha de clases y de la emancipación, así como la disensión teórica y metodológica de Marini frente a otras perspectivas. Con este capítulo, se espera contribuir con el desarrollo de esa teoría para el análisis actual de la formación dependiente y de sus transformaciones más recientes, que, a pesar del reconocimiento de la teoría de Marini, insisten en hacer resucitar mitos curiosos, como es el caso de la creencia en alguna fracción burguesa que estaría comprometida con un proyecto de "nación" o de la creencia en un «desarrollo capitalista nacional o autónomo» que, por consiguiente, garantizaría la superación de la condición dependiente por medio de las institucionalidades burguesas.

\section{Referencias}

Amaral, M. y Carcanholo M. (2008). Acumulaçâo capitalista e exército industrial de reserva: conteúdo da superexploraçáo do trabalho nas economias dependentes. Revista de Economia, (34), 163-181.

Carcanholo, M. (2013). (Im)precisiones acerca de la categoría superexplotación de la fuerza de trabajo. Razón y Revolución (25), 91-124.

Cardoso, F H. (2001). Comentario sobre los conceptos de sobrepoblación relativa y marginalidad [1970]. En J. Nun (ed.), Marginalidad y exclusión social. Fondo de Cultura Educatica.

Cardoso, F. H. y Serra J. (1978). As desventuras da dialética da dependência. Estudos CEBRAP, (23), 33-80.

Castro, N. A. (1983). Ejército de reserva: su especificidad y comportamiento político en el desarrollo del capitalismo en Brasil (tesis de grado de doctor en Sociología). Universidad Nacional Autónoma de México.

Engels, F. (2010). A situaçấo da classe trabalhadora na Inglaterra. Boitempo Editorial. Felix, G. (2018). Circulación y superexplotación del trabajo. Sociología del trabajo (92), 87-105. Felix, G. (2019). Mobilidade e superexploraçâo do trabalho: o enigma da circulaçâa. FAPESP/ Lamparina Editora. 
Felix, G. (2020). Super-circulation: towards a political economy of platformisation. Critical Sociology, 46(7-8),1221-1232.

Felix, G. (2021a). O Capital selvagem: mobilidade e superexploração do trabalho. Revista Latinoamericana de Antropología del Trabajo, (10), enero-abril.

Felix, G. (2021b). Aportes da teoria marxista da dependência para a análise da agropecuária e da indústria da mineraçâo. Revista Brasileira de Estudos Urbanos e Regionais, 23, jan-dez.

Felix, G. y Guanais, J. (coords.). (2018). Superexploração do trabalho no século XxI: debates contemporâneos. Editorial Praxis.

Felix, G. y Sotelo Valencia, A. (2019). A superexploração do trabalho: uma perspectiva da precariedade e da condição proletária a partir da teoria da dependência. Astrolabio, (23), 1-26.

Katz, C. (2017). Aciertos y problemas de la superexplotación. http://katz.lahaine.org/b2-img/ ACIERTOSYPROBLEMASDELASUPEREXPLOTACIN.pdf.

Kufakurinani, U. et al. (eds.). (2017). Dialogues on development (vol. 1, dependency). Institute for New Economic Thinking.

Marini, R. M. (1974). Subdesarrollo y revolución [1969]. Siglo XXI Editores.

Marini, R. M. (2008). Dialéctica de la dependencia [1973]. En C. Martins (ed.), América Latina, dependencia y g'lobalización. Siglo del Hombre/Clacso.

Marini, R. M. (2008a). En torno a dialéctica de la dependencia (postscriptum) [1973]. En C. Martins (ed.), América Latina, dependencia y g'lobalización. Siglo del Hombre/Clacso.

Marini, R. M. (1976). El reformismo y la contrarrevolución: estudios sobre Chile. Ediciones Era.

Marini, R. M. (1976 $)$. La reforma agraria en América Latina (Comentarios a la intervención de Michel Gutelman). Cuadernos Agrarios, (4). http://www.marini-escritos.unam.mx/281_ reforma_agraria.html

Marini, R. M. (1977). La acumulación capitalista mundial y el subimperialismo, Cuadernos Politicos (12), abril-junio.

Marini, R. M. (1977a). Estado y crisis en Brasil. Cuadernos Políticos, (13), julio-septiembre.

Marini, R. M. (1978). Las razones del neodesarrollismo (respuesta a F. H. Cardoso y J. Serra). Revista Mexicana de Sociología, XL, número extraordinario.

Marini, R. M. (1979). El ciclo del capital en la economía dependiente. En U. Oswald (ed.), Mercado y dependencia. Nueva Imagen.

Marini, R. M. (1979a). Plusvalía extraordinaria y acumulación de capital. Cuadernos Políticos (20), abril-junio.

Marini, R. M. (2012). Elementos para un balance histórico de treinta años de izquierda revolucionaria en América Latina [1989]. En P. Rivas (ed.). Ruy Mauro Marini. Editorial IAEN.

Marini, R. M. (s. f.). Memoria. http://www.marini-escritos.unam.mx/001_memoria_marini_ port.html

Marx, K. (2011). Grundrisse. Editorial Boitempo.

Marx, K. (2013). O capital. Livro I. Editorial Boitempo.

Nun, J. (2001). La teoría de la masa marginal [1969]. En J. Nun (ed.). Marginalidad y exclusión social. Fondo de Cultura Económica.

Osorio, J. (1979). Acumulación y ejército industrial de reserva en las economías dependientes. En Oswald (ed.), Mercado y dependencia. Editorial Nueva Imagen. 
Osorio, J. (2017). Teoría marxista de la dependencia sin superexplotación. Una propuesta de desarme teórico para avanzar. http://marxismoyrevolucion.org/wp-content/uploads/2017/09/ Teor\%C3\%ADa-marxista-de-la-dependencia-sin-superexplotación.pdf.

Smith, J. (2016). Imperialism in the twenty-first century: globalization, super-exploitation and capitalism's final crisis. Monthly Review Press.

Sotelo, A. (2016). The future of work: super-exploitation and social precariousness in the $21^{\text {st }}$ Century. Brill.

Sotelo, A. (2017). Crítica a la crítica de Katz a Marini: ¿una teoría de la dependencia sin superexplotación? Mejor una teoría de la dependencia con superexplotación revisitada y actualizada. http://rebelion.org/docs/235669.pdf 
